\title{
Migraine frequency and risk of cardiovascular disease in women
}

a 院

Tobias Kurth, MD, ScD

Markus Schürks, MD, MSc

Giancarlo Logroscino, $\mathrm{MD}, \mathrm{PhD}$

Julie E. Buring, $S c D$

Address correspondence to Dr. Tobias Kurth, INSERM Unit 708-Neuroepidemiology, Hôpital de la Pitié-Salpêtrière, 47 Boulevard de l'Hôpital, 75651 Paris Cedex 13, France tkurth@rics.bwh.harvard.edu

\section{ABSTRACT}

Background: Migraine has been associated with risk of cardiovascular disease (CVD). Data on the association between migraine frequency and CVD are sparse.

Methods: Prospective cohort study of 27,798 US women aged $\geq 45$ years, who were free of $C V D$, and for whom we had information on lipids and migraine frequency. We categorized migraine frequency as < monthly, monthly, and $\geq$ weekly. Incident CVD was confirmed after medical record review.

Results: Of the 3,568 women with active migraine at baseline, 75.3\% reported a migraine frequency of $<$ monthly, $19.7 \%$ monthly, and $5.0 \% \geq$ weekly. During 11.9 years of follow-up, 706 CVD events occurred. Compared with women without migraine, the multivariable-adjusted hazard ratios (HRs) (95\% confidence intervals) among active migraineurs for CVD were 1.55 (1.22-1.97), 0.65 (0.311.38 ), and $1.93(0.86-4.33)$ for an attack frequency of < monthly, monthly, and $\geq$ weekly, respectively. The association between migraine frequency and CVD was only apparent among migraineurs with aura. Among those, the multivariable-adjusted HRs for women with a migraine frequency < monthly ranged from 1.81 (1.30 -2.50) for coronary revascularizations to $2.43(1.58-3.74)$ for myocardial infarction. For women with active migraine with aura and migraine frequencies of $\geq$ weekly, we only found significant increased risk of ischemic stroke (HR $=4.25$ [1.36-13.29]).

Conclusions: In our data, the association between migraine and cardiovascular disease varies by migraine frequency. Significant associations were only found among women with migraine with aura. Ischemic stroke was the only outcome associated with a high-attack frequency while a low-attack frequency was associated with any vascular event. Low number of outcome events should caution the interpretation. Neurology ${ }^{\circledR} 2009 ; 73: 581-588$

\section{GLOSSARY}

$\mathbf{C I}=$ confidence interval; $\mathbf{C V D}=$ cardiovascular disease; $\mathbf{H R}=$ hazard ratio; $\mathbf{M I}=$ myocardial infarction; $\mathbf{W H S}=$ Women's Health Study.

Migraine is a primary, chronic-intermittent headache disorder that affects a large proportion of the population, predominantly women. ${ }^{1}$ It is characterized by recurrent headache attacks, which typically are located unilaterally, of pulsating pain quality, and of moderate to severe intensity. Migraine attacks are often accompanied by sensitivity to light and sound, vomiting, or nausea. In some patients, usually prior to headache onset, transient neurologic symptoms occur known as migraine aura, ${ }^{2}$ most often affecting the visual field.

A large body of literature supports an association between migraine and ischemic stroke ${ }^{3-9}$ that is in most studies limited to patients with migraine with aura. Recent findings from prospective cohort studies further suggest an association between migraine with aura and any vascular event, including

\section{Editorial, page 576}

e-Pub ahead of print on June 24, 2009, at www.neurology.org.

From the Division of Preventive Medicine (T.K., M.S., J.E.B.), Department of Medicine, Brigham and Women's Hospital, Harvard Medical School, Boston; Department of Epidemiology (T.K., J.E.B.), Harvard School of Public Health, Boston; INSERM Unit 708-Neuroepidemiology (T.K.) and University Pierre et Marie Curie (T.K.), Paris, France; and the Department of Neurology and Psychiatry (G.L.), School of Medicine, University of Bari, Italy. The Women's Health Study is supported by grants from the National Heart, Lung, and Blood Institute (HL-043851 and HL-080467) and the National Cancer Institute (CA-47988). The research for this work was supported by grants from the Donald W. Reynolds Foundation, the Leducq Foundation, and the Doris Duke Charitable Foundation. The sponsors of the study played no role in design or conduct of the study; collection, management, analysis, or interpretation of the data; or preparation, review, or approval of the manuscript.

Disclosure: Author disclosures are provided at the end of the article. 
myocardial infarction (MI). ${ }^{10-12}$ However, the biologic mechanisms linking migraine with ischemic vascular events have yet to be established. Potential mechanisms that increase the risk for cardiovascular disease (CVD) among migraineurs include prothrombotic effects, liability to vascular risk factors, impaired vascular reactivity, specific gene variants, congenital heart defects, migraine-specific treatments, or a direct involvement of the migraine pathophysiology. ${ }^{13,14}$

Moreover, it remains unclear whether migraine frequency is associated with risk of CVD. Results of previous case-control studies suggest that the risk for ischemic stroke further increases with rising attack frequency among women with migraine with aura. ${ }^{5,9} \mathrm{In}$ formation whether migraine frequency is associated with increased risk of major ischemic vascular events, including MI, is lacking. We thus aimed to evaluate whether the association between migraine and CVD differs according to migraine frequency in a large cohort of US female health professionals.

METHODS Study population. This was a prospective cohort study among participants in the Women's Health Study (WHS), a completed trial designed to test the benefits and risks of low-dose aspirin and vitamin $\mathrm{E}$ in the primary prevention of CVD and cancer among apparently healthy women. The design and results have been described in detail previously. ${ }^{15,16}$ Briefly, a total of 39,876 US female health professionals aged $\geq 45$ years at study entry (1992 to 1995) and without a history of CVD, cancer, or other major illnesses were randomly assigned to active aspirin (100 mg on alternate days), active vitamin E (600 IU on alternate days), both active agents, or both placebos. Baseline information was self-reported and was collected by a mailed questionnaire that asked about a large number of vascular risk factors and lifestyle variables. All participants provided written informed consent and the Institutional Review Board and Brigham and Women's Hospital approved the WHS. Twice in the first year and yearly thereafter, participants were sent follow-up questionnaires asking about study outcomes, personal characteristics, medical history, and health habits. For this analysis, we included follow-up information from study entry through March 31, 2007. As of this date, follow-up was $97 \%$ complete.

Before randomization, blood samples were collected in tubes containing EDTA from 28,345 participating women and stored in vapor phase liquid nitrogen $\left(-170^{\circ} \mathrm{C}\right)$. Samples were analyzed for lipids and a panel of inflammatory biomarkers. Total cholesterol was assayed with the use of reagents from Roche Diagnostics (Basel, Switzerland) and analyses could be performed on 27,939 of the blood samples.

Assessment of migraine. Participants were asked on the baseline questionnaire: "Have you ever had migraine headaches?" and "In the past year, have you had migraine headaches?" From this information, we categorized women into "no migraine his- tory" and "any history of migraine." Furthermore, we distinguished between "active migraine," which includes women with self-reported migraine in the year prior to completing the baseline questionnaire, and "prior migraine," which includes women who reported ever having had a migraine but none in the year prior to completing the questionnaire. Participants who reported active migraine were asked about migraine-specific features, including migraine frequency. Answer categories for migraine frequency included $<6$ times per year, every other month, monthly, weekly, and daily. From this information, we a priori recategorized migraine frequency into $<$ monthly, monthly, and $\geq$ weekly.

In previous studies of the WHS, ${ }^{10,17}$ we have shown good agreement with 1988 International Headache Society criteria for migraine. ${ }^{18}$ Specifically, we showed that among WHS participants who provided a blood sample and reported active migraine, $83.5 \%$ fulfilled all but one International Classification of Headache Disorders-I criteria (code 1.7, migrainous disorder) and 46.6\% fulfilled all IHS criteria for migraine (code 1.1, migraine without aura)..$^{10}$ In addition, we have shown ${ }^{19}$ that in a subsample of the WHS, over $87 \%$ of women with self-reported active migraine could be diagnosed with migraine without aura $(71.5 \%)$ or probable migraine without aura (16.2\%) according to International Classification of Headache Disorders-II criteria. ${ }^{20}$

Participants who reported active migraine were further asked whether they had an "aura or any indication a migraine is coming." Responses were used to classify women who reported active migraine into active migraine with aura and active migraine without aura.

Cardiovascular events. All participants were followed for the occurrence of the endpoint major CVD, a combined endpoint defined as the first of any of these events: nonfatal MI, nonfatal ischemic stroke, or ischemic cardiovascular death. In addition, we evaluated any first MI, ischemic stroke, coronary revascularization procedure (includes reports of coronary artery bypass grafts or percutaneous coronary angioplasty), and angina. There were too few cases of cardiovascular death to perform meaningful stratified analyses. Medical records were obtained for all reported cardiovascular endpoints except angina and reviewed by an Endpoints Committee of physicians. Nonfatal stroke was confirmed if the participant had a new focal-neurologic deficit of sudden onset that persisted for $>24$ hours and was then classified as ischemic, hemorrhagic, or undefined with excellent interobserver agreement. ${ }^{21}$ For this report, we only included ischemic stroke cases, and participants with other stroke subtypes were censored at the time of event. The occurrence of MI was confirmed if symptoms met World Health Organization criteria and if the event was associated with abnormal levels of cardiac enzymes or abnormal electrocardiograms. Deaths were confirmed by autopsy reports, death certificates, medical records, and information obtained from next of kin or family members and classified according to its specific cause.

Statistical analyses. Of the 27,939 women with completed blood assays, we excluded 79 with missing information on migraine status, 20 women who reported angina prior to receiving the baseline questionnaire, and 42 with missing information on migraine frequency, leaving 27,798 women free of CVD or angina at study entry for this analysis. We compared the baseline characteristics of participants with respect to migraine frequency status using analysis of covariance for continuous measurements, adjusting for age. We used direct standardization to adjust categorical variables for age in 5-year increments. 
Table 1 Baseline characteristics according to migraine frequency in the Women's Health Study $(n=27,798)$

\begin{tabular}{|c|c|c|c|c|c|c|}
\hline Mean age, y (SE) & $54.9(0.05)$ & $55.5(0.18)$ & $53.0(0.14)$ & $52.0(0.27)$ & $53.1(0.53)$ & $<0.001$ \\
\hline Mean body mass index, $\mathrm{kg} / \mathrm{m}^{2}$ (SE) & $25.9(0.03)$ & $26.1(0.13)$ & $26.2(0.10)$ & $25.6(0.19)$ & $25.5(0.37)$ & 0.006 \\
\hline Mean total cholesterol, mg/dL ${ }^{\ddagger}$ (SE) & $211.4(0.27)$ & $214.8(1.05)$ & $213.3(0.79)$ & $212.0(1.55)$ & $210.6(3.05)$ & 0.006 \\
\hline History of hypertension,,$\%$ & 24.6 & 30.2 & 25.8 & 24.4 & 31.5 & $<0.001$ \\
\hline \multicolumn{7}{|l|}{ Smoking, \% } \\
\hline Never & 51.2 & 50.5 & 54.1 & 56.1 & 57.8 & $<0.001$ \\
\hline Past & 37.0 & 35.4 & 35.8 & 36.3 & 33.6 & \\
\hline Current & 11.8 & 14.1 & 10.1 & 7.6 & 8.6 & \\
\hline \multicolumn{7}{|l|}{ Alcohol consumption, \% } \\
\hline Rarely/never & 43.5 & 45.0 & 46.8 & 49.0 & 56.9 & $<0.001$ \\
\hline $1-3$ drinks/mo & 13.1 & 14.2 & 14.7 & 12.7 & 17.1 & \\
\hline 1-6 drinks/wk & 32.7 & 30.5 & 30.5 & 30.5 & 21.2 & \\
\hline$\geq 1 \mathrm{drink} / \mathrm{d}$ & 10.8 & 10.3 & 8.0 & 7.8 & 4.8 & \\
\hline \multicolumn{7}{|l|}{ Physical activity, \% } \\
\hline Rarely/never & 37.0 & 39.2 & 39.0 & 38.5 & 39.9 & $<0.001$ \\
\hline$<1 /$ wk & 19.2 & 20.1 & 21.8 & 19.7 & 19.0 & \\
\hline $1-3 / w k$ & 32.3 & 29.5 & 29.3 & 30.2 & 34.1 & \\
\hline$\geq 4 / w k$ & 11.6 & 11.3 & 9.9 & 11.6 & 7.1 & \\
\hline \multicolumn{7}{|l|}{ Postmenopausal hormone therapy, \% } \\
\hline Never & 49.6 & 46.0 & 42.2 & 45.3 & 33.9 & $<0.001$ \\
\hline Past & 8.7 & 10.4 & 9.5 & 7.0 & 8.2 & \\
\hline Current & 41.7 & 43.6 & 48.3 & 47.7 & 58.0 & \\
\hline $\begin{array}{l}\text { Family history of myocardial infarction } \\
\text { before age } 60 \mathrm{y}, \%\end{array}$ & 11.4 & 12.4 & 12.6 & 11.4 & 9.0 & 0.30 \\
\hline
\end{tabular}

"Women with history of migraine but no active migraine in the year before completing the baseline questionnaire. ${ }^{t} p$ Values from analysis of covariance for continuous or Mantel-Haenszel test for categorical variables. 程o convert cholesterol values to millimoles per liter, multiply by 0.02586 .

s'Systolic blood pressure $\geq 140 \mathrm{~mm} \mathrm{Hg}$, diastolic blood pressure $\geq 90 \mathrm{~mm} \mathrm{Hg}$, physician-diagnosed hypertension, or antihypertensive treatment.

We used age-adjusted and multivariable-adjusted Cox proportional hazards models to calculate hazard ratios (HRs) and $95 \%$ confidence intervals (CIs) of the association between migraine frequency categories and CVD outcomes. In the multivariable models, we adjusted for age (continuous), history of hypertension (defined as systolic blood pressure $\geq 140 \mathrm{~mm} \mathrm{Hg}$, diastolic blood pressure $\geq 90 \mathrm{~mm} \mathrm{Hg}$, or physician diagnosis of hypertension, or antihypertensive treatment), body mass index $\left(<25,25-29.9, \geq 30 \mathrm{~kg} / \mathrm{m}^{2}\right)$, smoking status (never, past, current), total cholesterol (quartiles), postmenopausal hormone use (never, past, current), and family history of MI prior to age 60 (yes, no). We incorporated a missing value indicator variable if the number of women with missing information was $\geq 100$ or otherwise reassigned values of the reference group or past-user group, as applicable. Additional adjustment for alcohol consumption and exercise levels did not substantially change the effect estimates. Further, adjusting for randomized treatment assignments did not lead to different results.

In further analyses, we evaluated whether the association of migraine frequency and CVD outcomes was modified by migraine aura status. We evaluated statistically significant effect modification by using the likelihood ratio test. All analyses were performed using
SAS version 9.1 (SAS Inc, Cary, NC); $p$ values were two-tailed and a $p<0.05$ was considered statistically significant.

RESULTS Of the 27,798 women in this analysis, $5,083(18.3 \%)$ reported any history of migraine, of whom 3,568 (70.2\%) reported active migraine. Among active migraineurs, $75.3 \%$ reported a migraine frequency of $<$ monthly, $19.7 \%$ monthly, and $5.0 \% \geq$ weekly. In table 1 , we summarize the association between migraine frequency and baseline characteristics. Women with a migraine frequency of at least weekly tended to have lower cholesterol values, smoked less and drank less alcohol, and were more likely to have a history of hypertension and to have a history of postmenopausal hormone use as women with a migraine frequency of less then monthly.

During a mean of 11.9 years of follow-up (329,704 person-years), a total of 706 major CVD 
Table 2 Associations between migraine frequency and cardiovascular disease (CVD) in the Women's Health Study $(n=27,798)$

\begin{tabular}{|c|c|c|c|c|c|}
\hline & \multirow[b]{2}{*}{$\begin{array}{l}\text { No history of } \\
\text { migraine } \\
(n=22,715) \text { : } \\
\text { Referent }\end{array}$} & \multirow[b]{2}{*}{$\begin{array}{l}\text { History of migraine* } \\
\text { ( } n=1,515): \\
\text { HR }(95 \% \text { Cl) }\end{array}$} & \multicolumn{3}{|c|}{ Active migraine, frequency } \\
\hline & & & $\begin{array}{l}<\text { Monthly } \\
\text { (n =2,685): } \\
\text { HR (95\% Cl) }\end{array}$ & $\begin{array}{l}\text { Monthly } \\
\text { ( } n=703): \\
\text { HR }(95 \% \mathrm{Cl})\end{array}$ & $\begin{array}{l}\geq \text { Weekly } \\
\text { ( } n=180): \\
\text { HR }(95 \% \mathrm{Cl})\end{array}$ \\
\hline Major CVD $(n=706)$ & $n=567$ & $\mathrm{n}=48$ & $\mathrm{n}=78$ & $n=7$ & $\mathrm{n}=6$ \\
\hline Age-adjusted & 1.00 & $1.22(0.91-1.63)$ & $1.52(1.20-1.93)$ & $0.60(0.29-1.28)$ & $1.74(0.78-3.89)$ \\
\hline Multivariable-adjusted ${ }^{\ddagger}$ & 1.00 & $1.13(0.84-1.51)$ & $1.55(1.22-1.97)$ & $0.65(0.31-1.38)$ & $1.93(0.86-4.33)$ \\
\hline Myocardial infarction $(n=305)$ & $n=243$ & $\mathrm{n}=20$ & $\mathrm{n}=38$ & $\mathrm{n}=2$ & $\mathrm{n}=2$ \\
\hline Age-adjusted & 1.00 & $1.18(0.75-1.87)$ & $1.65(1.17-2.32)$ & $0.38(0.09-1.51)$ & $1.29(0.32-5.18)$ \\
\hline Multivariable-adjusted ${ }^{\ddagger}$ & 1.00 & $1.10(0.70-1.73)$ & $1.68(1.19-2.37)$ & $0.41(0.10-1.67)$ & $1.53(0.38-6.15)$ \\
\hline Ischemic stroke $(n=310)$ & $n=257$ & $\mathrm{n}=16$ & $\mathrm{n}=29$ & $\mathrm{n}=4$ & $\mathrm{n}=4$ \\
\hline Age-adjusted & 1.00 & $0.89(0.54-1.47)$ & $1.27(0.86-1.86)$ & $0.79(0.29-2.13)$ & $2.60(0.97-6.97)$ \\
\hline Multivariable-adjusted ${ }^{\ddagger}$ & 1.00 & $0.80(0.48-1.33)$ & $1.28(0.87-1.89)$ & $0.84(0.31-2.26)$ & $2.77(1.03-7.46)$ \\
\hline $\begin{array}{l}\text { Coronary revascularization }{ }^{\S} \\
(n=655)\end{array}$ & $n=530$ & $\mathrm{n}=48$ & $\mathrm{n}=67$ & $n=7$ & $\mathrm{n}=3$ \\
\hline Age-adjusted & 1.00 & $1.32(0.98-1.77)$ & $1.27(0.98-1.63)$ & $0.55(0.26-1.17)$ & $0.85(0.27-2.64)$ \\
\hline Multivariable-adjusted ${ }^{\ddagger}$ & 1.00 & $1.22(0.90-1.63)$ & $1.26(0.97-1.63)$ & $0.60(0.28-1.26)$ & $0.93(0.30-2.89)$ \\
\hline Angina $(n=418)$ & $n=322$ & $\mathrm{n}=41$ & $\mathrm{n}=48$ & $\mathrm{n}=4$ & $\mathrm{n}=3$ \\
\hline Age-adjusted & 1.00 & $1.84(1.33-2.55)$ & $1.49(1.10-2.02)$ & $0.52(0.19-1.40)$ & $1.39(0.45-4.33)$ \\
\hline Multivariable-adjusted ${ }^{\ddagger}$ & 1.00 & $1.70(1.23-2.36)$ & $1.48(1.09-2.02)$ & $0.56(0.21-1.51)$ & $1.53(0.49-4.78)$ \\
\hline
\end{tabular}

"Women with history of migraine but no active migraine in the year before completing the baseline questionnaire.

${ }^{+} A$ major CVD event was defined as the first of any of these events: nonfatal ischemic stroke, nonfatal myocardial infarction, or death from ischemic cardiovascular cause.

¥Adjusted for age, history of hypertension, smoking, body mass index, total cholesterol, postmenopausal hormone use, and family history of myocardial infarction before age $60 \mathrm{y}$.

$\S$ Includes reports of coronary artery bypass grafting or percutaneous coronary angioplasty.

events (nonfatal MI, nonfatal ischemic stroke, or death from ischemic CVD) occurred, which, taking into account the potential for multiple events in a single individual, included 305 first MIs, 310 ischemic strokes, and 151 CVD deaths. In addition, 655 women had coronary revascularization procedures and 418 women reported angina. In table 2, we summarize the age-adjusted and multivariable-adjusted association between migraine frequency and incident major CVD, MI, and ischemic stroke. Compared with women without a history of migraine, we found J-shaped associations between migraine frequency and major CVD, a finding driven by ischemic strokes. In multivariable-adjusted models, the HRs (95\% CIs) for major CVD were 1.55 (1.22-1.97; $p<0.001), 0.65(0.31-1.38 ; p=0.27)$, and 1.93 $(0.86-4.33 ; p=0.11)$ for a migraine frequency of $<$ monthly, monthly, and $\geq$ weekly, respectively. Women who reported a history of migraine but no active migraine in the year before completing the baseline questionnaire had a HR of $1.13(0.84-1.51$; $p=0.43)$. While we found increased relative risks for the outcomes MI and ischemic stroke in the highmigraine and low-migraine frequency group, the emphasis differed. Among women with active migraine, we observed the strongest association with $\mathrm{MI}$ in the lowest migraine frequency category $(\mathrm{HR}=1.68$; $95 \% \mathrm{CI}=1.19-2.37 ; p=0.003)$, while the strongest association for ischemic stroke was found in the high-migraine frequency group ( $\mathrm{HR}=2.77 ; 95 \%$ $\mathrm{CI}=1.03-7.46 ; p=0.04)$. We found no significant association between migraine frequency and coronary revascularization. For angina, we found the strongest association among women with prior migraine $(\mathrm{HR}=1.70 ; 95 \% \mathrm{CI}=1.23-2.36 ; p=$ $0.001)$ but also for women with active migraine and a migraine frequency of $<$ monthly $(\mathrm{HR}=1.48$; $95 \% \mathrm{CI}=1.09-2.02 ; p=0.01$ ).

When we took migraine aura status into account, we only observed a significant association between migraine frequency and CVD for women with migraine with aura (table 3; $p$ for interaction $=0.009$ ). We found strong and significant association with all ischemic vascular events among women with active migraine with aura with a low migraine frequency $(<$ monthly), ranging from a HR of $1.81(95 \% \mathrm{CI}=$ $1.30-2.50 ; p<0.001)$ for coronary revascularization to $2.43(95 \% \mathrm{CI}=1.58-3.74 ; p<0.001)$ for MI. For women with active migraine with aura and a migraine frequency of at least one per week, we only 


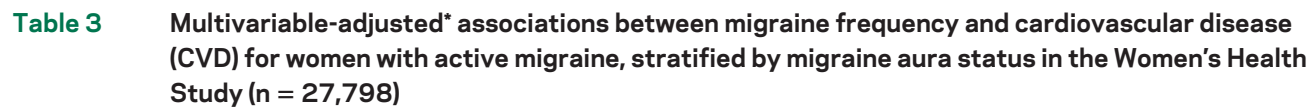

Migraine without aura $(n=2,140)$

Major $\mathrm{CVD}^{+}(\mathrm{n}=37)$

Myocardial infarction $(n=17)$

Ischemic stroke ( $n=15)$

Coronary revascularization ${ }^{\ddagger}(n=33)$

Angina $(n=27)$

Migraine with aura $(n=1,428)$

Major $\mathrm{CVD}^{+}(\mathrm{n}=54)$

Myocardial infarction $(n=25)$

Ischemic stroke $(n=22)$

Coronary revascularization $n^{\ddagger}(n=44)$

Angina $(n=28)$
Active migraine, frequency
$<$ Monthly

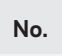

No.

No. $\mathrm{n}=1,590$

$30 \quad 1.02(0.71-1.48) \quad 4$

$151.14(0.67-1.92) \quad 1$

$110.84(0.46-1.53)$

28

22

$0.88(0.60-1.30)$

$1.16(0.75-1.80)$

$\mathrm{n}=1,095$

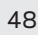

$2.28(1.70-3.07)$

2.43 (1.58-3.74)

1.90 (1.18-3.08)

1.81 (1.30-2.50)

$1.93(1.29-2.89)$

\section{Monthly}

No.

HR (95\% Cl)

$n=453$

0.61 (0.23-1.63)

$0.34(0.05-2.41)$

1.04 (0.33-3.27)

0.42 (0.13-1.29)

$0.68(0.22-2.12)$

$\mathrm{n}=250$

$0.72(0.23-2.25)$

$0.53(0.08-3.81)$

0.53 (0.07-3.77)

0.89 (0.33-2.39)

$0.37(0.05-2.65)$ $\geq$ Weekly

No. $\quad H R(95 \% \mathrm{Cl})$

$\mathrm{n}=97$

$3 \quad 1.89(0.61-5.88)$

1

$1.42(0.20-10.15)$

$1.36(0.19-9.69)$

$1.13(0.28-4.53)$

$1.92(0.48-7.71)$

$\mathrm{n}=83$

$1.98(0.64-6.17)$

$1.64(0.23-11.72)$

4.25 (1.36-13.29)

0.69 (0.10-4.88)

$1.09(0.15-7.76)$

Models include an indicator variable for women who reported a history of migraine but not active migraine in the year before completing the baseline questionnaire. Women without a history of migraine serve as the reference group. Effect estimates and number of events for women with a history of migraine or without a history of migraine are listed in table 2.

*Adjusted for age, history of hypertension, smoking, body mass index, total cholesterol, postmenopausal hormone use, and family history of myocardial infarction before age $60 \mathrm{y}$.

${ }^{+}$A major cardiovascular event was defined as the first of any of these events: nonfatal ischemic stroke, nonfatal myocardial infarction, or death from ischemic cardiovascular cause.

\#Includes reports of coronary artery bypass grafting or percutaneous coronary angioplasty.

found significant association with ischemic stroke $(\mathrm{HR}=4.25 ; 95 \% \mathrm{CI}=1.36-13.29 ; p=0.01)$.

DISCUSSION In this large, prospective cohort of women aged $\geq 45$ years, we found particular patterns of associations between migraine frequency among active migraineurs and specific CVD events. The association with major CVD appeared J-shaped with increased risks for $<$ monthly and $\geq$ weekly attacks, a pattern also seen for stroke. The association with MI and angina showed a U-shaped association pattern. When we stratified by migraine aura status, we found no significant associations between migraine frequency and any ischemic vascular event among women with migraine without aura. In contrast, we found strong and significant associations between low migraine frequency ( $<$ monthly) and major CVD among women with active migraine with aura. In addition, women with active migraine with aura with a migraine frequency of at least weekly had over fourfold increased risk for ischemic stroke but not for any other ischemic vascular event.

The association between migraine frequency and CVD has previously only been evaluated for the outcome ischemic stroke. One study compared 86 women aged 20-44 with ischemic stroke with 214 controls. ${ }^{5}$ The results showed that women with migraine with aura with an initial migraine frequency of $\geq 13$ per year had substantially increased risk of ischemic stroke $(\mathrm{OR}=10.4 ; 95 \% \mathrm{CI}=2.18-49.4)$ while those with a migraine frequency of $<13$ per year had an OR of 3.58 (95\% CI $=0.86-14.8)$. The results of this study also suggested that an increase in migraine frequency had stronger associations with ischemic stroke than a decrease in migraine frequency. ${ }^{5}$ Another study found no changes of the association between change in migraine frequency and stroke, but this was limited to women who used oral contraceptives. ${ }^{22}$

In a population-based case-control study among women aged 15 to 49 years, 386 cases of ischemic stroke were age-matched and ethnicity-matched with 614 controls and participants were classified according to migraine status and migraine aura status. ${ }^{9}$ Women with migraine with visual aura had increased risk of ischemic stroke that was only apparent for those with a migraine frequency of $>12$ per year (adjusted $\mathrm{OR}=1.7 ; 95 \% \mathrm{CI}=1.1-2.8$ ) but not for those with less frequent migraine $(\mathrm{OR}=0.9 ; 95 \%$ $\mathrm{CI}=0.6-1.4)$. In a previous report of the WHS, we found no association between migraine frequency and ischemic stroke but, because of the lower number of outcome events, we only could dichotomize migraine frequency in $<$ monthly and $\geq$ monthly. ${ }^{7}$ 
The association between migraine and silent infarcts as well as white matter lesions was investigated in the CAMERA study. In this population-based study from the Netherlands, the risk of infarcts in the posterior circulation territory increased with increasing migraine frequency $\left(p_{\text {trend }}<0.005\right) .{ }^{23}$ Compared with controls, the risk of infarcts was 9.3 times higher among participants with a migraine frequency of at least once per month. Similarly, a significant association between migraine and silent infarct-like lesions in the posterior circulation tended to be stronger with a higher migraine frequency $(\geq 1$ per month). ${ }^{24}$

Our results of substantially increased risk for ischemic stroke for women with active migraine with aura and a high migraine frequency are in agreement with the results of the prior studies. It remains unclear whether the association between migraine frequency and ischemic stroke in these studies increases again with lower migraine frequency, because migraine frequency could only be dichotomized.

Our data have several strengths, including the large number of participants and outcome events, confirmed vascular events after medical record review, with the exception of angina, standardized assessment of migraine, and the relative homogenous nature of our cohort, which may reduce confounding.

Several limitations should be considered when interpreting our data. First and most importantly, while the number of outcome events was large in the overall group, there were only limited outcome events in subgroups. Thus, as in previous studies 5, $9,23,24$ and despite statistical significance, our effect estimates carry some degree of uncertainty, which should caution strong conclusions. Second, we only assessed migraine frequency at baseline, thus we could not evaluate the association of change of patterns of migraine frequency with risk of CVD, an association suggested by another study. ${ }^{5}$ Third, migraine and migraine features were self-reported. While we have shown good agreement with 1988 International Headache Society criteria for migraine $^{10}$ and excellent agreement between selfreported migraine and International Classification of Headache Disorders-II-based migraine classification in the WHS,${ }^{19}$ misclassification is possible. However, due to the prospective nature of our data, such misclassification would likely yield an underestimation of observed associations. Fourth, despite control for a number of potential confounders in our analysis, residual confounding is possible since our study is observational. Finally, participants in our study were all $\geq 45$ years of age, health professionals, and mostly Caucasian. Thus, generalizability to other populations may be limited.
While there are several hypothesis why migraine may be linked with CVD, ${ }^{14}$ the mechanisms explaining this association remain unclear. Increasing evidence suggests that this link is particularly strong among migraineurs with aura. For ischemic stroke, smoking and oral contraceptive use have been identified as additional modifying factors. ${ }^{4,6,9,22,25}$ Migraine frequency may be another modifying factor, although associations may be complex and differential patterns may exist for specific CVD events. Diametric associations have also been found for the link between migraine with aura and specific CVD events according to vascular risk status ${ }^{26}$ and for the $677 \mathrm{C}>\mathrm{T}$ polymorphism in the methylenetetrahydrofolate reductase gene. ${ }^{27,28}$ It may be plausible that migraine frequency interacts with other modifying factors, such as age or vascular risk. There is evidence that patients with active migraine have less severe arteriosclerosis, ${ }^{29,30}$ which may suggest that vascular health plays a role in migraine occurrence. It has to be established whether changes in migraine frequency associate with vascular functions and whether other modifying factors help to explain links between migraine frequency and CVD events. We had too few outcome events in subgroups to further evaluate this hypothesis. While overweight and obesity do not seem to be associated with migraine overall, body mass index has consistently been associated with migraine frequency ${ }^{31-33}$ and two studies suggest that this association may be U- or J-shaped. ${ }^{32,33}$ Since we have controlled for body mass index in our analysis, we believe that the association between migraine frequency and CVD is not strongly influenced by obesity.

If migraine per se would increase the risk of CVD, one would expect that an increase in the frequency would lead to further risk increase. The pattern of association of our data only supports this hypothesis for ischemic stroke. However, migraine frequency may not necessarily be a measure of migraine severity. In addition, since associations between migraine and vascular events are only found for migraine with aura in most studies, aura frequency rather than migraine frequency may be a better marker of further increased risk of CVD; such information was not available in our data or other studies. Further, since the 1-year prevalence of migraine and migraine frequency decreases with increasing age, migraine and migraine frequency patterns over time may also be of interest in predicting future CVD events.

Taken together, migraine with aura per se may not be sufficient to increase the risk of CVD but additional factors seem to be necessary. These additional factors include vascular risk factors, ${ }^{3,9,26}$ migraine frequency, ${ }^{5,9}$ gene variants, ${ }^{27,28}$ use of oral contraceptives, ${ }^{22,25}$ or smoking. ${ }^{4,9}$ Further, there is evidence that the mecha- 
nisms leading to ischemic stroke or coronary events among migraineurs with aura differ. ${ }^{26,27}$ Thus, future studies are warranted to evaluate the association of migraine, migraine subforms, and migraine patterns with risk of vascular events.

\section{AUTHOR CONTRIBUTIONS}

T.K. had full access to all the data in the study and takes responsibility for the integrity of the data, the accuracy of the data analysis, and the decision to submit for publication. T.K. conceived and designed the study, analyzed the data, as well as drafted the manuscript. All authors interpreted the data, critically revised the draft for important intellectual content, and gave final approval of the manuscript to be published. J.E.B. obtained funding.

\section{ACKNOWLEDGMENT}

The authors thank the participants in the Women's Health Study for thei commitment and cooperation and the Women's Health Study staff for their assistance.

\section{DISCLOSURE}

Dr. Kurth has received funding for travel from the Restless Legs Syndrome Foundation; received honoraria for educational lectures from Genzyme and Pfizer; serves as a consultant to i3 Drug Safety and World Health Information Science Consultants, LLC, two for-profit companies evaluating drug safety and surveillance; and receives research support from Merck (IISP ID 35437) to study associations between migraine and cardiovascular disease and from the NIH (HL091880 [PI]; NS061836 [PI]). Dr. Schuerks received an honorarium from L.E.K. Consulting for a telephone survey and had received research support from the German Research Foundation (Deutsche Forschungsgemeinschaft [DFG]). Dr. Logroscino serves as Associate Editor of Neuroepidemiology. Dr. Buring receives research support from Dow Corning, Amgen, and the NIH (CA047988 [PI]; HL080467 [PI]).

Received December 3, 2008. Accepted in final form April 6, 2009.

\section{REFERENCES}

1. Lipton RB, Bigal ME. The epidemiology of migraine. Am J Med 2005;118 suppl 1:3S-10S.

2. Goadsby PJ, Lipton RB, Ferrari MD. Migraine: current understanding and treatment. N Engl J Med 2002;346:257-270.

3. Henrich JB, Horwitz RI. A controlled study of ischemic stroke risk in migraine patients. J Clin Epidemiol 1989;42: 773-780.

4. Tzourio C, Iglesias S, Hubert JB, et al. Migraine and risk of ischaemic stroke: a case-control study. BMJ 1993;307: 289-292.

5. Donaghy M, Chang CL, Poulter N. Duration, frequency, recency, and type of migraine and the risk of ischaemic stroke in women of childbearing age. J Neurol Neurosurg Psychiatry 2002;73:747-750.

6. Etminan M, Takkouche B, Isorna FC, Samii A. Risk of ischaemic stroke in people with migraine: systematic review and meta-analysis of observational studies. BM] 2005;330:63-65.

7. Kurth T, Slomke MA, Kase CS, et al. Migraine, headache, and the risk of stroke in women: a prospective study. Neurology 2005;64:1020-1026.

8. Stang PE, Carson AP, Rose KM, et al. Headache, cerebrovascular symptoms, and stroke: the Atherosclerosis Risk in Communities Study. Neurology 2005;64:1573-1577.

9. MacClellan LR, Giles WH, Cole J, et al. Probable migraine with visual aura and risk of ischemic stroke: The Stroke Prevention in Young Women Study. Stroke 2007;38:24382445.
10. Kurth T, Gaziano JM, Cook NR, Logroscino G, Diener $\mathrm{HC}$, Buring JE. Migraine and risk of cardiovascular disease in women. JAMA 2006;296:283-291.

11. Kurth T, Gaziano JM, Cook N, et al. Migraine and risk of cardiovascular disease in men. Arch Intern Med 2007;167: 795-801.

12. Liew G, Wang JJ, Mitchell P. Migraine and coronary heart disease mortality: a prospective cohort study. Cephalalgia 2007;27:368-371.

13. Tietjen GE. Migraine as a systemic disorder. Neurology 2007;68:1555-1556.

14. Vargas BB, Dodick DW, Wingerchuk DM, Demaerschalk BM. Migraine with and without aura and risk for cardiovascular disease. Curr Atheroscler Rep 2008;10:427-433.

15. Rexrode KM, Lee IM, Cook NR, Hennekens CH, Buring JE. Baseline characteristics of participants in the Women's Health Study. J Womens Health Gend Based Med 2000; 9:19-27.

16. Ridker PM, Cook NR, Lee IM, et al. A randomized trial of low-dose aspirin in the primary prevention of cardiovascular disease in women. N Engl J Med 2005;352:12931304.

17. Bensenor IM, Cook NR, Lee IM, Chown MJ, Hennekens $\mathrm{CH}$, Buring JE. Low-dose aspirin for migraine prophylaxis in women. Cephalalgia 2001;21:175-183.

18. Classification and diagnostic criteria for headache disorders, cranial neuralgias and facial pain: Headache Classification Committee of the International Headache Society. Cephalalgia 1988;8 suppl 7:1-96.

19. Schurks M, Buring JE, Kurth T. Agreement of selfreported migraine with ICHD-II criteria in the Women's Health Study. Cephalalgia 2009 (in press).

20. The International Classification of Headache Disorders: 2nd edition. Cephalalgia 2004;24 suppl 1:9-160.

21. Atiya M, Kurth T, Berger K, Buring JE, Kase CS. Interobserver agreement in the classification of stroke in the Women's Health Study. Stroke 2003;34:565-567.

22. Chang CL, Donaghy M, Poulter N. Migraine and stroke in young women: case-control study: The World Health Organisation Collaborative Study of Cardiovascular Disease and Steroid Hormone Contraception. BMJ 1999; 318:13-18.

23. Kruit MC, van Buchem MA, Hofman PA, et al. Migraine as a risk factor for subclinical brain lesions. JAMA 2004; 291:427-434.

24. Kruit MC, Launer LJ, Ferrari MD, van Buchem MA. Infarcts in the posterior circulation territory in migraine: the population-based MRI CAMERA study. Brain 2005;128: 2068-2077.

25. Oral contraceptives and stroke in young women: associated risk factors. JAMA 1975;231:718-722.

26. Kurth T, Schurks M, Logroscino G, Gaziano JM, Buring JE. Migraine, vascular risk, and cardiovascular events in women: prospective cohort study. BMJ 2008;337:a636.

27. Schurks M, Zee RY, Buring JE, Kurth T. Interrelationships among the MTHFR $677 \mathrm{C}>\mathrm{T}$ polymorphism, migraine, and cardiovascular disease. Neurology 2008;71:505-513.

28. Pezzini A, Grassi M, Del Zotto E, et al. Migraine mediates the influence of C677T MTHFR genotypes on ischemic stroke risk with a stroke-subtype effect. Stroke 2007;38: 3145-3151.

29. Ahmed B, Bairey Merz CN, McClure C, et al. Migraines, angiographic coronary artery disease and cardiovascular outcomes in women. Am J Med 2006;119:670-675. 
30. Schwaiger J, Kiechl S, Stockner H, et al. Burden of atherosclerosis and risk of venous thromboembolism in patients with migraine. Neurology 2008;71:937-943.

31. Scher AI, Stewart WF, Ricci JA, Lipton RB. Factors associated with the onset and remission of chronic daily headache in a population-based study. Pain 2003;106:81-89.
32. Bigal ME, Tsang A, Loder E, Serrano D, Reed ML, Lipton RB. Body mass index and episodic headaches: a populationbased study. Arch Intern Med 2007;167:1964-1970.

33. Winter AC, Berger K, Buring JE, Kurth T. Body mass index, migraine, migraine frequency and migraine features in women. Cephalalgia 2009;29:269-278.

\section{Apply Today for Clinical Research Training Fellowships}

Fast track your career with an AAN Foundation 2010 Clinical Research Training Fellowship. Six types of opportunities are available to benefit researchers with salaries and tuition stipends:

- AAN Foundation Clinical Research Training Fellowship in Headache NEW IN 2010!

- AAN Foundation Clinical Research Training Fellowship in Stroke NEW IN 2010!

- AAN Foundation Clinical Research Training Fellowships MULTIPLE AVAILABLE!

- AAN Foundation Practice Research Training Fellowship

- AAN Foundation/MGFA Clinician-Scientist Development Three-year Award (cosponsored by the Myasthenia Gravis Foundation of America and the AAN Foundation) NEW IN 2010!

- NMSS-AAN Foundation Multiple Sclerosis Clinician Scientist Development Award (cosponsored by the National Multiple Sclerosis Society and the AAN Foundation) (August 14 deadline)

Apply online at www.aan.com/fcrtf by October 1, 2009.

\section{Earn Practice Management CME with AAN Audio Conferences}

The Academy is helping members take some of the confusion out of coding with a four-part series.

The 2009 Coding Audio Conferences will review proper coding in common circumstances, helping participants to code with greater precision. Upon completion, physician participants will receive 1 CME credit per call, up to $4 \mathrm{CME}$ credits total. Non-neurologists (e.g., practice managers) will receive a certificate of completion redeemable for credits. Special pricing is available when you register for more than one call and several people can listen in from one office-making these sessions particularly cost effective as well as educational.

For details on savings and to register, visit www.aan.com/codingcme. 